\title{
Posterior Surgery Alone in the Treatment of Post-traumatic Kyphosis by Posterior Column Osteotomy, Spondylodesis, Instrumentation, and Vertebroplasty
}

\author{
Farzad Omidi-Kashani ${ }^{1}$, Ebrahim Ghayem Hasankhani ${ }^{1}$, \\ Mohamed Hosein Ebrahimzadeh ${ }^{2}$, Amir Reza Kachooei ${ }^{2}$, Hosein Heidari ${ }^{1}$ \\ ${ }^{1}$ Orthopedic Department, Orthopedic Research Center, Emam Reza Hospital, Mashhad University of Medical Sciences, Mashhad, Iran \\ ${ }^{2}$ Orthopedic Department, Orthopedic Research Center, Ghaem Hospital, Mashhad University of Medical Sciences, Mashhad, Iran
}

Study Design: Retrospective study.

Purpose: To determine if posterior surgery alone can satisfactorily treat post-traumatic kyphosis (PTK).

Overview of Literature: One of the worst complications of vertebral fractures is PTK. The type of surgery and approach to treat a symptomatic and refractory PTK is a challenging issue in spinal surgery, and yet, there is no specific treatment algorithm.

Methods: From August 2003 to September 2010, we collected 26 cases (male to female ratio, 2.25; mean age, 31.9ะ9.7 years and follow-up period of $42.4 \pm 8.1$ months) with PTK treated by posterior column osteotomy, spondylodesis, instrumentation and cement vertebroplasty in one stage posterior surgery. PTK angle, Oswestry Disability Index (ODI), visual analogue scale (VAS), and subjective satisfaction from surgery were used to determine the results. We used a student $t$ test for analyzing the data before and after surgery. Results: In our patients, T11 and L1 had the highest incidence of vertebral fractures. The results indicated that in PTK, ODI, and VAS were significantly improved this surgery. Solid fusion occurred in $96.2 \%$ of patients with $3.2^{\circ} \pm 2.1^{\circ}$ loss of correction. A total of $84.6 \%$ of patients have satisfaction level of excellent and good.

Conclusions: Posterior surgery alone with posterior column osteotomy, vertebroplasty, posterior spinal fusion and instrumentation can effectively treat symptomatic PTK.

Keywords: Spinal fusion; Osteotomy; Kyphosis, Post traumatic

\section{Introduction}

One of the worst complications of vertebral fractures is post-traumatic kyphosis (PTK). This posture puts the paravertebral muscles in inappropriate position and ultimately, could lead to fatigue and pain, especially in cases with focal kyphosis of more than $30^{\circ}$ [1-3]. In the mild forms of PTK, physical therapy associated with muscle strengthening exercises may be helpful in relieving the pain and discomfort, but in severe cases however, surgical intervention is sometimes necessary. The type of surgery to treat PTK is a challenging issue in spinal surgery, and

Received Oct 22, 2012; Revised Jan 14, 2013; Accepted Feb 12, 2013

Corresponding author: Farzad Omidi-Kashani

Orthopedic Department, Orthopedic Research Center, Emam Reza Hospital, Mashhad University of Medical Sciences, 913791-3316, Mashhad, Iran

Tel: +98-915-514-9248, Fax: +98-511-859-5023, E-mail: omidif@mums.ac.ir 
currently, there is no clear treatment plan.

As this complication has a delayed nature, a contracted and fibrotic tissue is formed at the anterior part of the injured spine and an attenuated posterior musculoligamentous complex at the posterior; therefore, the logical surgical approach for this complication seems to be comprised of two surgical stages. First, an anterior spinal release and fusion (preferably with a strut bone graft or cage) should be carried out and then the spinal alignment should be more corrected and stabilized by posterior fusion and instrumentation. Some authors advocated anterior surgery alone for its treatment and claimed that in the absence of significant osteoporosis or posterior ligamentous disruption, anterior approach permits the removal of pathological contracted tissues, anterior releasing, and kyphosis correction, insertion of a strut graft or cage, as well as stabilization of the spine with a rigid instrumentation $[4,5]$.

Because of a plenty of disadvantages associated with anterior approaches, in this study, we omitted it by adding the Ponte osteotomy (posterior element wedge resection) [6], and vertebroplasty to the routine posterior spinal fusion and instrumentation to improve the clinical results; however, as we will explain later, according to the exclusion criteria, the severe cases have been eliminated from the study. The aim of this retrospective study is to evaluate the clinical and radiological results of this posterior surgery alone in the treatment of symptomatic and refractory PTK.

\section{Materials and Methods}

From August 2003 to September 2010, we retrospectively collected the cases with post-traumatic kyphosis, treated with posterior column osteotomy, spondylodesis, instrumentation and cement vertebroplasty in one stage posterior surgery. The minimum follow-up period allowed for the participants was 24 months. Initially, 29 patients fulfilled our criteria to be included in the study, but 3 were excluded due to insufficient follow-up data. Therefore, we eventually studied 26 patients in whom the male to female ratio was 2.25 (18 to 8 ) and the mean age and follow-up period were $31.9 \pm 9.7$ and $42.4 \pm 8.1$, respectively.

Before surgery, all the patients with PTK had been treated conservatively for at least 3 months. We excluded the cases with associated osteoporosis, significant neurologic deficit (myelopathy vs. radiculopathy) necessitated anterior decompression, age $>60$ years, $\mathrm{PTK}$ angle $>50^{\circ}$ relative to the normal regional alignment (this point will be explained further in the next section), vertebral body collapse of more than two third of the original height, significant associated underlying disease (uncontrollable diabetes mellitus, immunodeficiency disorder, etc.) and those patients who could not give up smoking in the critical perioperative period. The first authors (F.O-K and E.G.H) completely explained the pros and cons of the surgery to the patients preoperatively, and all of them signed the informed consent forms.

Normal regional sagittal alignment was assessed based on thoracic kyphosis, which usually starts at T1-T2 (averaging $+1^{\circ}$ at that segment) and increases at each segment until the apex (at T6-T7 where it reaches to $+5^{\circ}$ ) and then gradually decreases until the straight thoracolumbar junction is reached. Lumbar lordosis usually begins at L1-L2 (averaging $-4^{\circ}$ ) and gradually increases caudally [7].

Preoperatively, standing anteroposterior and lateral plain radiographs of the thoracolumbar spine and magnetic resonance imaging scanning were taken. PTK was ascertained by drawing the lines parallel with the superior end plate of the intact adjacent upper vertebra and the lower end plate of the intact adjacent lower vertebra. Patients' disability and pain were assessed by Oswestry Disability Index (ODI) questionnaire version 2.1 and a 0 to 10 pain intensity numerical rating scale (visual analogue scale, VAS), respectively $[8,9]$.

Patient satisfaction from the surgery was also determined subjectively as excellent (ready to do again the same procedure for the same problem), good (the surgery improved the status but did not merit all the patient's expectations), fair (the patient's status did not changed with surgery), and poor (when the surgery has worsened the patient's condition).

\section{Surgical technique}

After induction of general anesthesia, the patient was placed in prone position on two transverse rolls with maximum attention to avoid flat back posturing. The abdomen was freely dangled to avoid excessive epidural bleeding. Midline longitudinal incision and subperiosteal paravertebral muscles dissection were routinely performed. Vertebral level identification was carried out by intraoperative fluoroscopy. Ponte osteotomy [6] was 
conducted in the involved segments, and then pedicular screws were inserted under the biplane fluoroscopic control. These screws were inserted in two levels up and down of the injured vertebra. In the middle and lower lumbar regions, which are normally located anterior to the gravity plumb line, the screws were only inserted in one level up and down of the intended vertebra. Posterior decompression was carried out in those cases with the associated nerve root compression (Fig. 1). Bilateral cement vertebroplasty with polymethylmethacrylate (PMMA) was conducted under direct pedicular vision and fluoroscopic control to enhance the anterior and middle columns' compressive endurance. Two lordotic molded longitudinal rods were inserted and strengthened by a transverse load connector in long constructs (Fig. 2). After the wound was irrigated, the posterior osseous surface of the involved vertebrae was carefully and completely decorticated by a fine power burr and a plenty of auto- and allogenic bone graft were added to obtain a thick fusion mass layer. Then, the wound was closed in the anatomical planes on a suction drain.

\section{Postoperation}

The patient was ambulated the day after with a lumbosacral or thoracolumbosacral orthosis. Suction drain was removed 24 to 48 hours after the surgery, and then usually, the patient was discharged from the hospital on that day, except unusual conditionings. Brace was removed after 3 months. We visited the patient at 2 weeks, 1.5, 3, 6 , and 12 months after surgery, and with annual intervals thereafter. After 6 months, the questionnaires were completed at each visit, but our criterion for data analysis was the forms completed in the last session. We did not routinely use computerized tomography scanning to determine spondylodesis and our criteria for a solid union in this study were observing the bridging bone between the involved vertebrae, absence of motion in dynamic (flexion-extension) views, absence of any implant failure or clinically significant pain.

\section{Statistics}

Descriptive statistics were calculated as the mean \pm standard deviation or as the median and inter quartile range (IQR) according to the distributions of the continuous variables. We used student $\mathrm{t}$ test for analyzing the data before and after the surgery. Statistical significance was determined using a 5\% significance level $(p<0.05)$. Statistical analysis was performed using SPSS ver. 11.5 (SPSS Inc., Chicago, IL, USA).

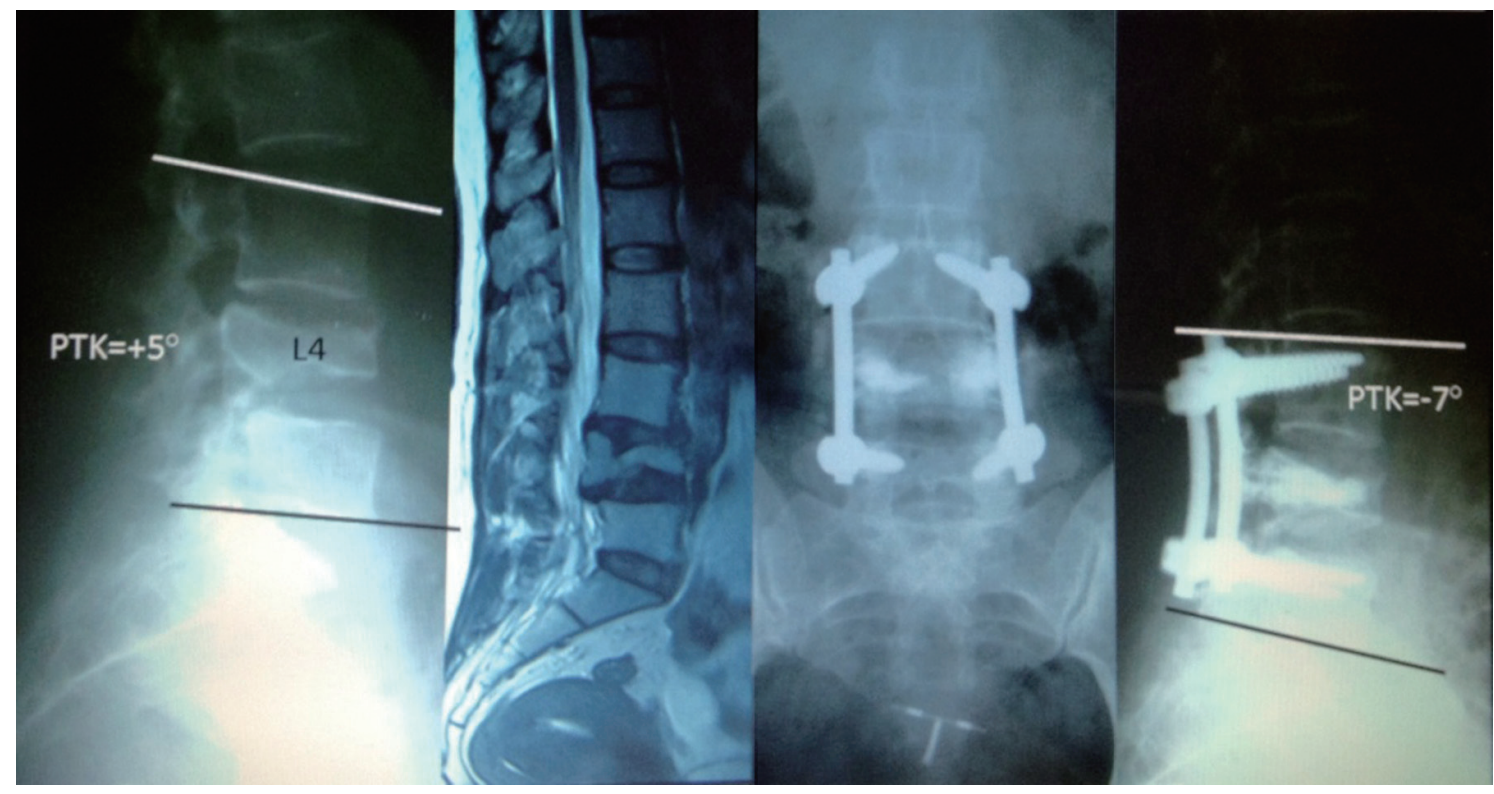

Fig. 1. A 46-year-old female with a history of motor vehicle accident 10 months ago, presented with refractory low back pain and lower limbs paresthesia. She was operated by L4 laminectomy, L4 and L5 nerve roots decompression, bilateral L4 vertebroplasty, posterior L3-L5 spinal fusion and instrumentation. PTK, post-traumatic kyphosis. 
Table 1. Incidence of vertebral fractures in our patients

\begin{tabular}{lc} 
Vertebra & Incidence (\%) \\
T10 & 7.7 \\
T11 & 19.2 \\
T12 & 15.4 \\
L1 & 19.2 \\
L2 & 11.5 \\
L3 & 7.7 \\
L4 & 7.7 \\
T12, L1 & 7.7 \\
\hline T11, T12 & 3.8 \\
\hline Total & 100 \\
\hline
\end{tabular}

\section{Results}

As expected, the most common site for PTK in our patients was thoracolumbar junction. Table 1 shows the fre- quency of fracture in the vertebrae. T11 and L1 had the highest incidence of vertebral fractures.

Table 2 shows the preoperative and postoperative PTK angle, ODI, and VAS. The postoperative scores were calculated based on the latest follow up visit (minimum 24 months). The table indicates that all the three parameters were significantly improved by surgery $(p<0.05)$. Solid fusion occurred in $96.2 \%$ of patients (25 of 26). Patients with pseudarthrosis were clinically asymptomatic, but presented with one pedicular screw breakage at the distal part of the construct discovered during routine follow-up visit. The mean loss of correction between immediate and the latest follow-up visits was $3.2^{\circ} \pm 2.1^{\circ}$.

A total of $84.6 \%$ of patients expressed the satisfaction level as either excellent or good. Other ones have moderate level of satisfaction. In no cases poor result has occurred. There was no complications at all in 61.5\%; in remaining patients, the complications included asymptomatic cement leakage ( 7 cases; $26.9 \%$ ), superficial

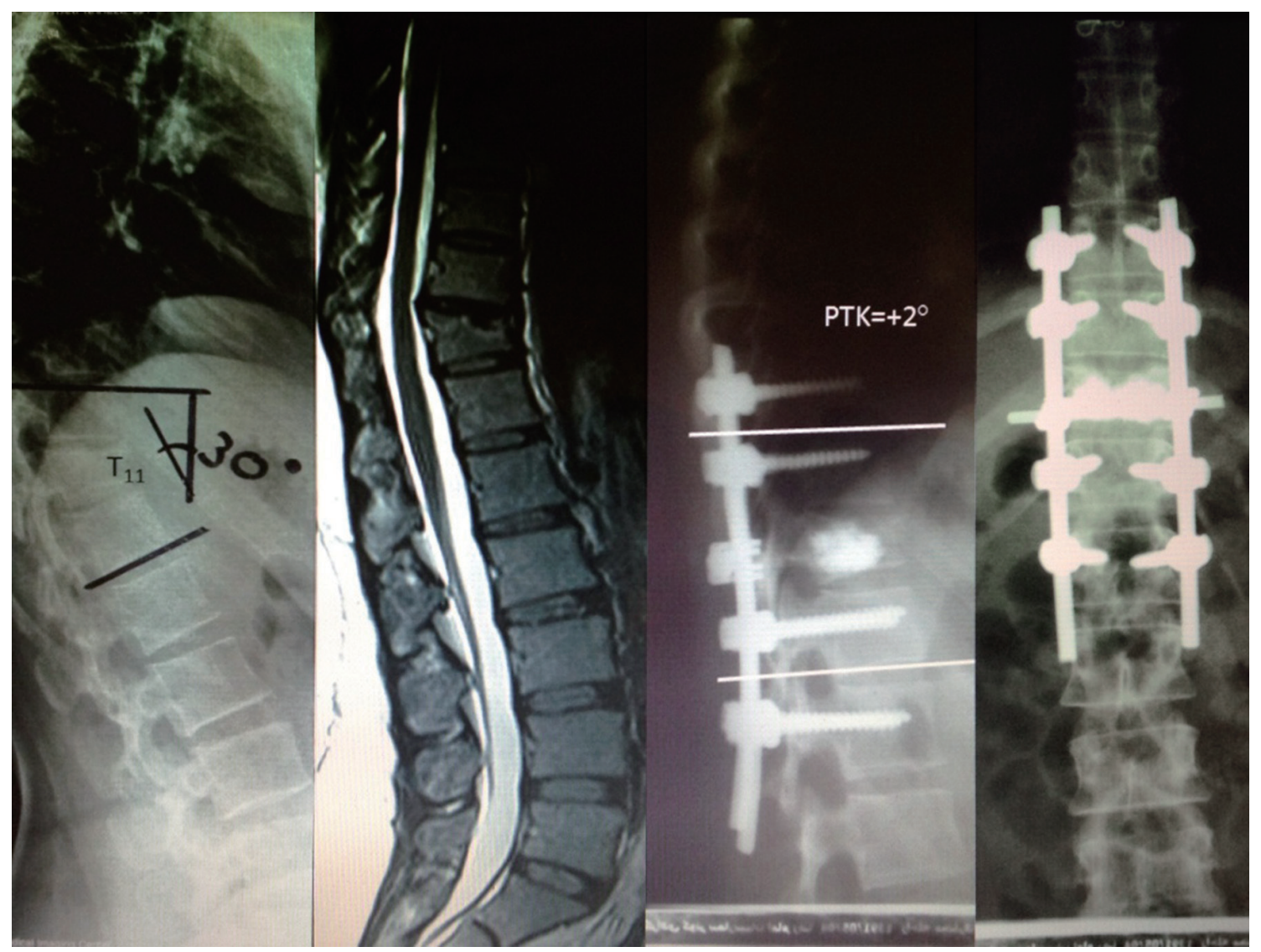

Fig. 2. A 30-year-old female was presented with disabling back pain pertaining to the car-pedestrian accident 6 months ago when she was 7 months pregnant. She did not take any imaging scan on that date. Spinal osteotomy, fusion and instrumentation plus cement vertebroplasty were all conducted with posterior only approach. PTK changed from $+30^{\circ}$ to $+2^{\circ}$ in the latest follow-up visit 28 months later. PTK, post-traumatic kyphosis. 
Table 2. Clinical and radiological status of our patients before and after surgery

\begin{tabular}{lccc}
\multicolumn{1}{c}{ Index } & Preoperative & Postoperative & $p$-value \\
\hline Post-traumatic kyphosis $\left({ }^{\circ}\right)$ & $26.6 \pm 9.58$ & $2.0 \pm 4.02$ & $<0.0001$ \\
Oswestry Disability Index & $41.6 \pm 6.55$ & $5.1 \pm 2.84$ & $<0.0001$ \\
Visual analogue scale & $7.0 \pm 1.41$ & $1.3 \pm 0.87$ & $<0.0001$ \\
\hline
\end{tabular}

wound infection ( 2 cases; $7.7 \%$ ), and pedicular screw breakage (1 case; $3.8 \%)$. There wasn't any significant correlation between the degree of PTK correction and the degree of ODI and VAS improvement $(p$-value $=0.532$ and 0.813 , respectively).

\section{Discussion}

Painful kyphotic angulation of the traumatic spine is a well known late condition among the spine injured patients that primarily have been treated inappropriately. The most common primary spinal injuries responsible for producing this condition are severe types of bust fractures and flexion distraction injuries [10]. Nonetheless, the surgeon should not forget about the other traumaticrelated underlying etiologies, like deep infection, osteonecrosis of the vertebral body (Kummell's disease), or neuropathic spinal arthropathy (Charcot spine) [10-12]. The primary injuries may have been initially neglected, treated conservatively or even surgically operated. Symptomatic patients are usually presented with increasing pain, deformity or neurologic deficit [13].

In spite of significant advances in spine surgical issues in recent decades, there are still abundant controversies concerning the preferred surgical treatment of PTK. Studies showed that the neurologic deficit in the setting of PTK has two main reasons. While increasing kyphosis may tent the spinal cord and create or aggravate the neurologic deficit, most of this complains come from the developing post-traumatic syringomyelia [14].

Surgical indications quoted for symptomatic PTK include refractory pain, progressive deformity, and neurologic deficit. Although multiple approaches have been carried out to treat PTK $[5,15,16]$, in the cases with insufficient anterior structural support, posterior only approaches will be condemned to fail. Therefore, some kind of anterior support seemed to be necessary.

As we intended to correct the PTK with posterior approach alone, some kind of spinal osteotomy should be included. Because most of our patients were young (with relative normal intervertebral discs) and our deformities were moderate, Ponte osteotomy seemed to be a more appropriate and safe modality. This type of osteotomy was first explained in 1984 by Ponte and Vero [6] for the correction of flexible Scheuermann's kyphosis in skeletally mature patients. Fear of implant failure to maintain longterm kyphosis correction by posterior approach alone, has led us to do anterior column supporting with cement augmentation (vertebroplasty). Another reason for using vertebroplasty in these relatively young patients back to the general philosophy of cement usage; the pain associated with vertebral fracture may be a result of slow healing or nonunion of the fracture with consequent instability and micromotion or pain can be caused by irritation of intraosseous nerve endings that cement with its structural support and exothermic effect can effectively treat both of this problems [17].

Zhang et al. [18] treated 26 non-osteoporotic patients with PTK by a single posterior trans-pedicle+disc osteotomy technique. They did not find any severe complications and they could averagely correct the spinal deformity from $22.3^{\circ}$ to $2.2^{\circ}$. Although the type of osteotomy in this study was different from our study, the study design, patient selection criteria (age and deformity), and surgical results are very similar to and supports our result. All patients in this study completed at least 3 years of follow-up, and VAS and ODI averagely improved from preoperative 8.6 and 62.5 to final postoperative 2.2 and 16.2, respectively. They recommended this modified type of osteotomy for treating thoracolumbar PTK.

Klostermann et al. [19] prospectively treated 28 cases with PTK and postinflammatory kyphosis by transpedicular wedge resection osteotomy (posterior approach alone) and reported satisfactory clinical and radiological results. Similar to our study, they evaluated their patients with a PTK angle, VAS, and ODI scoring pre- and postoperatively. They reported the mean kyphosis correction of $28^{\circ}$ (range, $14^{\circ}-44^{\circ}$ ), in which a $7^{\circ}$ of loss was noticed 
at the latest follow-up visit after one year. In comparing with the study we conducted, the kyphosis correction is similar but a loss of correction is significant in this study $\left(7.0^{\circ}\right.$ vs. $\left.3.2^{\circ}\right)$.

Although we did not see any neurologic complication among our patients, the reported rate in the literature ranged from $0 \%$ to $20 \%[20,21]$. The risk factors commonly cited for this include type of post-traumatic deformity (kyphosis vs. scoliosis), type and technique of the osteotomy and instrumentation [10]. All of our wound infections were superficial and easily treated with local wound care and short-term oral antibiotic therapy but the incidence was somewhat higher relative to the reported rate in the literature (7.7\% compared with $0 \%-7 \%$ ) $[10,20,21]$.

In this study, we could not find a significant correlation between the degree of PTK correction and clinical improvement. We think the likely reason for this is due to the fact that we initially excluded the relatively severe cases of PTK from this study. Our study had two major weaknesses that need to be mentioned. First, the study design was retrospective not prospective and this type of study is prone to selection or information bias as a result of the retrospective nature of this study. Second, the number of patients in our study was relatively low. Therefore, to be able to do a more reliable recommendation, a prospective study should be performed on more patients.

\section{Conclusions}

Although the number of cases we studied was relatively low, it can be claimed that posterior surgery alone with posterior column osteotomy, vertebroplasty, posterior spinal fusion and instrumentation can effectively treat symptomatic thoracolumbar PTK.

\section{Conflict of Interest}

No potential conflict of interest relevant to this article was reported.

\section{References}

1. Malcolm BW, Bradford DS, Winter RB, Chou SN. Post-traumatic kyphosis. A review of forty-eight surgically treated patients. J Bone Joint Surg Am 1981;63:891-9.
2. Gertzbein SD. Scoliosis Research Society: multicenter spine fracture study. Spine (Phila Pa 1976) 1992;17:528-40.

3. Roberson JR, Whitesides TE Jr. Surgical reconstruction of late post-traumatic thoracolumbar kyphosis. Spine (Phila Pa 1976) 1985;10:307-12.

4. El-Sharkawi MM, Koptan WM, El-Miligui YH, Said GZ. Comparison between pedicle subtraction osteotomy and anterior corpectomy and plating for correcting post-traumatic kyphosis: a multicenter study. Eur Spine J 2011;20:1434-40.

5. Benli IT, Kaya A, Uruc V, Akalin S. Minimum 5-year follow-up surgical results of post-traumatic thoracic and lumbar kyphosis treated with anterior instrumentation: comparison of anterior plate and dual rod systems. Spine (Phila Pa 1976) 2007;32:986-94.

6. Ponte A, Vero B. Surgical treatment of Scheuermann's hyperkyphosis. In: Winter R, editor. Progress in spinal pathology: kyphosis. Bologna: Aulo Gaggi; 1984. p.75-8.

7. Bernhardt M, Bridwell KH. Segmental analysis of the sagittal plane alignment of the normal thoracic and lumbar spines and thoracolumbar junction. Spine (Phila Pa 1976) 1989;14:717-21.

8. Fairbank JC, Pynsent PB. The Oswestry Disability Index. Spine (Phila Pa 1976) 2000;25:2940-52.

9. Wewers ME, Lowe NK. A critical review of visual analogue scales in the measurement of clinical phenomena. Res Nurs Health 1990;13:227-36.

10. Vaccaro AR, Silber JS. Post-traumatic spinal deformity. Spine (Phila Pa 1976) 2001;26:S111-8.

11. Young WF, Brown D, Kendler A, Clements D. Delayed post-traumatic osteonecrosis of a vertebral body (Kummell's disease). Acta Orthop Belg 2002;68:13-9.

12. Keene JS, Lash EG, Kling TF Jr. Undetected posttraumatic instability of "stable" thoracolumbar fractures. J Orthop Trauma 1988;2:202-11.

13. Schoenfeld AJ, Wood KB, Fisher CF, et al. Posttraumatic kyphosis: current state of diagnosis and treatment: results of a multinational survey of spine trauma surgeons. J Spinal Disord Tech 2010;23:e1-8.

14. Buchowski JM, Kuhns CA, Bridwell KH, Lenke LG. Surgical management of posttraumatic thoracolumbar kyphosis. Spine J 2008;8:666-77.

15. Lazennec JY, Neves N, Rousseau MA, Boyer P, Pascal-Mousselard H, Saillant G. Wedge osteotomy for 
treating post-traumatic kyphosis at thoracolumbar and lumbar levels. J Spinal Disord Tech 2006;19:48794.

16. Kirchmier RS, Denis F. Surgical considerations of posttraumatic spinal deformity. In: Cotler JM, Simpson JM, An HS, Siveri CP, editors. Surgery of spinal trauma. Philadelphia: Lippincott, Williams and Wilkins; 2000. p.317-32.

17. Siemionow K, Lieberman IH. Vertebral augmentation in osteoporosis and bone metastasis. Curr Opin Support Palliat Care 2007;1:323-7.

18. Zhang XS, Wang Y, Zhang YG, et al. Modified posterior closing wedge osteotomy in patients of posttraumatic thoracic lumbar kyphosis. Zhonghua Wai Ke
Za Zhi 2009;47:1383-6.

19. Klostermann CK, Hette K, Pflugmacher R. Transpedicular osteotomy with dorsal wedge osteotomy: treatment of post-traumatic or postinfection kyphotic malalignment of the thoraco-lumbar spine. Unfallchirurg 2009;112:1041-6.

20. Been HD, Poolman RW, Ubags LH. Clinical outcome and radiographic results after surgical treatment of post-traumatic thoracolumbar kyphosis following simple type A fractures. Eur Spine J 2004;13:101-7.

21. Heary RF, Bono CM. Pedicle subtraction osteotomy in the treatment of chronic, posttraumatic kyphotic deformity. J Neurosurg Spine 2006;5:1-8. 